\title{
The Sweeping-out of Dust by Radiation Pressure of Stars and Chemical Composition Peculiarities of Disc Galaxies
}

\author{
E. E. Sivkova ${ }^{a, *}$, D. S. Wiebe ${ }^{a}$, and B. M. Shustov ${ }^{a}$ \\ ${ }^{a}$ Institute of Astronomy, Russian Academy of Sciences, Moscow, 119017 Russia \\ *e-mail: sivkovae@gmail.com \\ Received October 11, 2020; revised December 30, 2020; accepted December 30, 2020
}

\begin{abstract}
We consider the drift of dust grains of various sizes and chemical compositions caused by the stellar radiation pressure in the vicinity of the Milky Way. When integrating the equations of motion, in addition to the radiation pressure, we consider the gravitational attraction from various components of the Galaxy and the gas drag. It has been shown that carbonaceous grains of medium sizes $(\sim 0.01 \mu \mathrm{m})$ are swept out of the galactic disc most effectively. Smaller dust grains are swept out to a substantially lesser extent, or they are not swept out at all. We also consider the motion of silicate dust grains, including those with porous structure. It has been shown that silicate grains experience a considerably weaker impact of the radiation pressure. The simulation result of their motion does not essentially depend on whether their porosity is accounted for or ignored. The total rate of the Galaxy's dust loss has turned out to be high-approximately $0.03 M_{\odot}$ per year, which is comparable to the effect produced by the other mechanisms ejecting heavy elements to the circumgalactic space. We discuss the potential of the sweeping of dust out of the Galaxy in formation of the radial metallicity gradient, as well as the prospects of detecting extensive dust structures in elliptical galaxies.
\end{abstract}

DOI: $10.1134 / \mathrm{S} 1063772921050061$

\section{INTRODUCTION}

Cosmic dust is one of the main components of the interstellar medium (ISM) in both our Galaxy and other similar galaxies. The main bulk of dust is concentrated in galactic discs; and observations show that the thickness of a dust disc is, as a rule, smaller than that of a stellar disc, while in a radial direction, the dust is observed even at larger distances than the stars [1-4]. However, substantial amounts of dust are often observed also at larger distances from the plane of symmetry of disc galaxies, in galactic halos. This is evidenced, for example, by vertical dark lanes branching away from galactic discs [5-8]. In this regard, the edge-on galaxy NGC 891 is among those studied most extensively. The authors of a series of papers $[6,9]$ detected numerous dark dust filaments, extending to the altitudes higher than $2 \mathrm{kpc}$, there. In the vicinity of the NGC 891 galactic disc, the mass of individual filaments is $\sim 10^{5} M_{\odot}$, while the total mass of extraplanar gas is $\sim 10^{8} M_{\odot}$, which amounts to $2 \%$ of the total mass of interstellar matter in this galaxy. The analysis of the Hubble Space Telescope observations of dust filaments in NGC 891 revealed that they are stretching to altitudes of about $1.5 \mathrm{kpc}$ perpendicular to the disc, which points to the link between the formation of dust structures and the dynamic phenomena in the direction perpendicular to the disc [10].
Observations of the absorption in the optical range yielded similar results for the other spiral galaxies [7, 11]. While individual dust structures are found at altitudes of the order of a few kiloparsecs, the signs of the dust-induced diffuse reddening are sometimes observed at distances of a few tens of kiloparsecs to a few megaparsecs $[12,13]$.

One more manifestation of the dust presence at high galactic altitudes is so-called ultraviolet (UV) halos. The detection of the extraplanar diffuse UV radiation in the galaxy NGC 891 was reported in papers $[14,15]$. The properties of this radiation suggest that there is a "thick" dust disc there (its vertical size is larger than that of a usual thin disc), where dust grains scatter the UV radiation of stars composed the galactic disc. In a paper [16], it is reported that analogous extensive regions of the diffuse UV radiation are observed around many almost edge-on galaxies at distances up to $20 \mathrm{kpc}$. The properties of this radiation are consistent with the presence of extraplanar dust grains that scatter the UV radiation of stars of the galactic disc.

Direct evidences of the dust presence at high galactic altitudes may be obtained from observations in the IR range. From the Infrared Space Observatory (ISO) data, Irwin and Madden [17] found the emission of polycyclic aromatic hydrocarbons (PAHs) in lengthy structures, extending for several kiloparsecs in the halo of the galaxy NGC 5907. Analogous structures 
were also found in the halo of the galaxy NGC 5529 [18]. Observations of the galaxy NGC 5775 at the Spitzer telescope show that the filamentary emission of PAH grains at a wavelength of $8 \mu \mathrm{m}$ is traced in this galaxy to $z \sim 5 \mathrm{kpc}$ [19]. The absorption peculiarity observed at a wavelength of $6565 \AA$ may be indirect evidence of the PAH presence in the halo of our Galaxy [20]. Dust observations in the halo of NGC 891 with the Herschel space telescope are described in a paper [21], where the scale height of the dust disc was estimated from the observations in a range of 70 to $250 \mu \mathrm{m}$ at approximately $1.5 \mathrm{kpc}$. Observations carried out at the Planck space observatory revealed the dust emission in the direction toward the North Polar Spur in our Galaxy [22].

In general, it can be taken for granted that dust is a typical component of halos in disc galaxies, which is observed at distances of at least a few kiloparsecs and the mass of which may amount to several percent of the total mass of dust in a galaxy. The interest to extraplanar dust is connected with the study of sources and dynamics of dust grains in galaxies, as well as with probable noises that may be introduced by this dust into different observations [23]. Meanwhile, there are still no clear answers what mechanism may take dust away to high altitudes and how much the properties of dust in galactic halos and thin discs differ. The estimates of typical sizes of dust grains in the circumgalactic medium differ. For example, in a paper [24], it was shown that the radius of carbonaceous dust grains in galactic halos varies approximately from 0.01 to $0.03 \mu \mathrm{m}$, which roughly coincides with the range for the ISM in our Galaxy. On the other hand, there are evidences that, in disc galaxies, the properties of dust grains in a halo and a disc may differ [19, 25].

To interpret the discussed observations, it is necessary to develop models describing the extraplanar dust genesis. Since dust grains can be formed at low temperature and high density of the gas, in which this process takes place, and these conditions are not realized outside galaxies, it is logical to assume that dust is formed within galaxies and swept out to the circumgalactic space. The most evident mechanism of the dust sweeping out of galaxies is the motion of dust under the influence of the galactic wind. This mechanism is actually efficient in the galaxies with active formation of stars and/or active nuclei [26-29]. However, extraplanar dust is also observed in the galaxies exhibiting a low rate of the star formation $[17,18]$. The wind may be only one of the mechanisms that sweeps dust out of a galaxy, since the spatial distributions of dust and gas in galactic halos may be different [30].

Sweeping dust out by the stellar radiation pressure is considered as an alternative mechanism in the literature. For the first time, this idea was expressed several decades ago [31-33]; and the sweeping of dust out of a disc galaxy has been simulated more than once [3438]. The authors of those studies usually come to con- clusion that the sweeping-out of dust by the stellar radiation pressure is typical of most disc galaxies and may result in the observed emerging of extraplanar dust, which is accompanied by ejecting a substantial amount of heavy elements from galactic discs.

Dust grains in the ISM do not remain unchanged (see, e.g., [39]). When moving in the ISM, they get to the regions of different properties and experience alterations. Consequently, when modeling the dust evolution, one should consider both the dynamic factors and the kinetics of dust grains. One of the first attempts of this kind was made in a paper [39]. For the recent quarter of a century, our knowledge on cosmic dust has substantially deepened, and it has become possible to correct and specify noticeably better the notions on the evolution of the dust component in disc galaxies. In this paper, we improve the model developed for the drift of dust under the influence of the stellar radiation pressure [40]. The model has been expanded to perform three-dimensional calculations of the motion of dust grains of different sizes and chemical composition; it also accounts for different initial coordinates, the stellar radiation pressure, the gravitation, and the interstellar gas drag. The influence of a dark halo was also included into the model. In Section 2, we explain the model of the dust sweeping out of the Galaxy and present the suppositions on the properties and sources of dust grains. In Section 3, we describe the main calculation results that characterize the motion of dust in the circumgalactic space. Astrophysical applications of the results are discussed in Section 4.

\section{THE MODEL OF THE DUST MOTION IN A GALAXY}

In the proposed model, the dynamics of dust in a galaxy is determined by three forces: the radiation pressure force, the gravitational attraction, and the drag force of interstellar gas. To calculate the forces of the radiation pressure and the gravity, the model for the distribution of the radiation field and the gravitational potential in the Galaxy and the model for the properties of dust grains are required. To calculate the gas drag force, it is also necessary to specify the properties of the medium through which the dust moves.

\subsection{Properties of Dust Grains}

In this paper, we consider dust grains of three types: graphite, silicate, and PAH. They differ by the density of matter and the optical properties.

The interaction of dust grains with radiation is determined by the processes of absorption, scattering, and polarization, which are characterized by the corresponding cross-sections. The efficiency of the radi- 

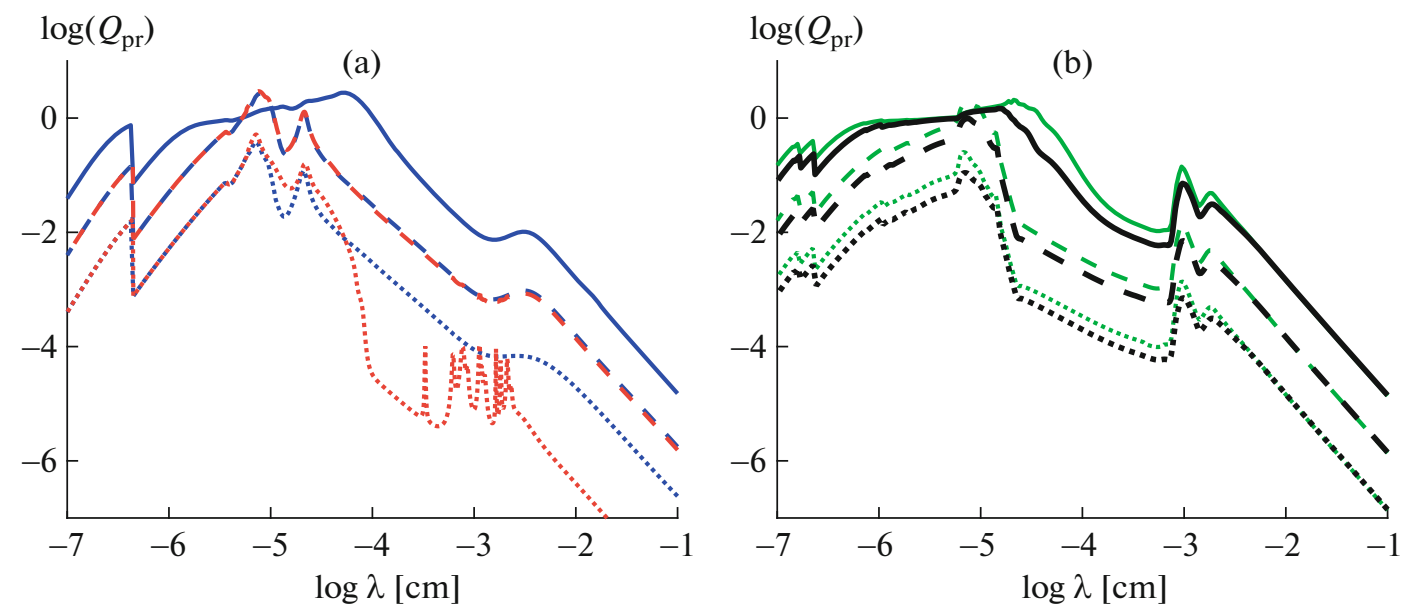

Fig. 1. The wavelength dependence of the radiation pressure efficiency factors $Q_{\mathrm{pr}}(v)$ for graphite (a) and silicate (b) grains. The data for grains with sizes of 10,100 , and $1000 \AA$ (or $0.1 \mu \mathrm{m}$ ) are shown by dotted, dashed, and solid curves, respectively. (a) The dependences for graphite and PAH grains are presented by blue and red curves, respectively. (b) The dependences for densely packed and porous (the porosity is $50 \%$ ) silicate grains are presented by green and black curves, respectively.

ation momentum transfer to a dust grain at a frequency $\checkmark$ is described by the radiation pressure efficiency

$$
Q_{\mathrm{pr}}(v)=Q_{\mathrm{abs}}(v)+Q_{\mathrm{sca}}(v)(1-\langle\cos \theta(v)\rangle),
$$

where $Q_{\text {abs }}(v)$ and $Q_{\text {sca }}(v)$ are the dimensionless coefficients, which are the ratios of the absorption and scattering cross-sections to the geometric cross-section of a grain, and the quantity $\langle\cos \theta(v)\rangle$ characterizes its scattering properties. The values of $Q_{\mathrm{abs}}(\mathrm{v}), Q_{\mathrm{sca}}(\mathrm{v})$, and $\langle\cos \theta(v)\rangle$ for graphite and silicate grains are assumed according to a paper [41], where they were calculated for a wide range of sizes and frequencies. It is worth noting that the ideas about the structure and chemical composition of so-called carbonaceous dust grains vary. In the following, we will call these grains graphite from the point of the optical properties and the density of matter assumed. The optical properties of PAHs were taken from [42]. ${ }^{1}$

When calculating the radiation pressure force, we used the values of $Q_{\mathrm{pr}}$, which were obtained with formula (1) and averaged according to the Planck spectral distribution of the energy for the radiation source temperature assumed (see below).

The optical properties of the considered dust grains are illustrated in Figs. 1 and 2. Figure 1a presents the efficiency factors of the radiation pressure in dependence on the wavelength for graphite grains of three sizes and PAH grains of two sizes. Figure $1 \mathrm{~b}$ presents the analogous dependences for silicate grains of different sizes. Hereafter, the data for grains with sizes of 10 , 100 , and $1000 \AA$ are shown by dotted, dashed, and solid curves, respectively. In the diagrams, the color indicates the chemical composition (structure) of

\footnotetext{
${ }^{1}$ https://www.astro.princeton.edu/draine/dust/dust.diel.html
}

grains: blue, red, green, and black curves correspond to graphite, PAH, silicate, and porous silicate dust, respectively. It is seen from the diagrams that the value of $Q_{\mathrm{pr}}(v)$ for large dust grains is an order of magnitude higher than that for medium-size grains, which, in turn, an order of magnitude higher than the value for small dust grains. It is also seen that the values of $Q_{\mathrm{pr}}$ for carbonaceous grains with a radius of $100 \AA$ and larger are almost independent of the assumption on their structure (PAH or graphite). For small grains, the value of $Q_{\mathrm{pr}}(\mathrm{v})$ at wavelengths of $10^{-4}-10^{-1} \mathrm{~cm}$ noticeably depends on the material of a grain; however, this value is substantially lower than $Q_{\mathrm{pr}}(v)$ for the same grains in the optical range.

The structure of silicate grains may also vary. Specifically, we cannot exclude that these grains in the ISM are porous [43]; i.e., their mean density may be smaller than that of densely packed grains. In this case, the mass of a grain of a specified size decreases; consequently, the attraction of the Galaxy will be weaker, which may facilitate the sweeping-out of a dust grain to the circumgalactic space. However, the optical properties of porous grains will be different as well. In Fig. $1 \mathrm{~b}$, the wavelength dependences of the radiation pressure efficiency factors for porous and dense silicate grains of three sizes are shown. To calculate the quantity $Q_{\mathrm{pr}}(v)$ for porous grains, the model from a paper [44] was used. It is seen from Fig. $1 \mathrm{~b}$ that the porosity of a dust grain somewhat diminishes the value of $Q_{\mathrm{pr}}(\mathrm{v})$; however, even in this case, the accounting for the structure will not be critical, since the value of $Q_{\mathrm{pr}}(v)$ decreases approximately by the same percentage as the mass of a grain.

ASTRONOMY REPORTS Vol. $65 \quad$ No. $5 \quad 2021$ 

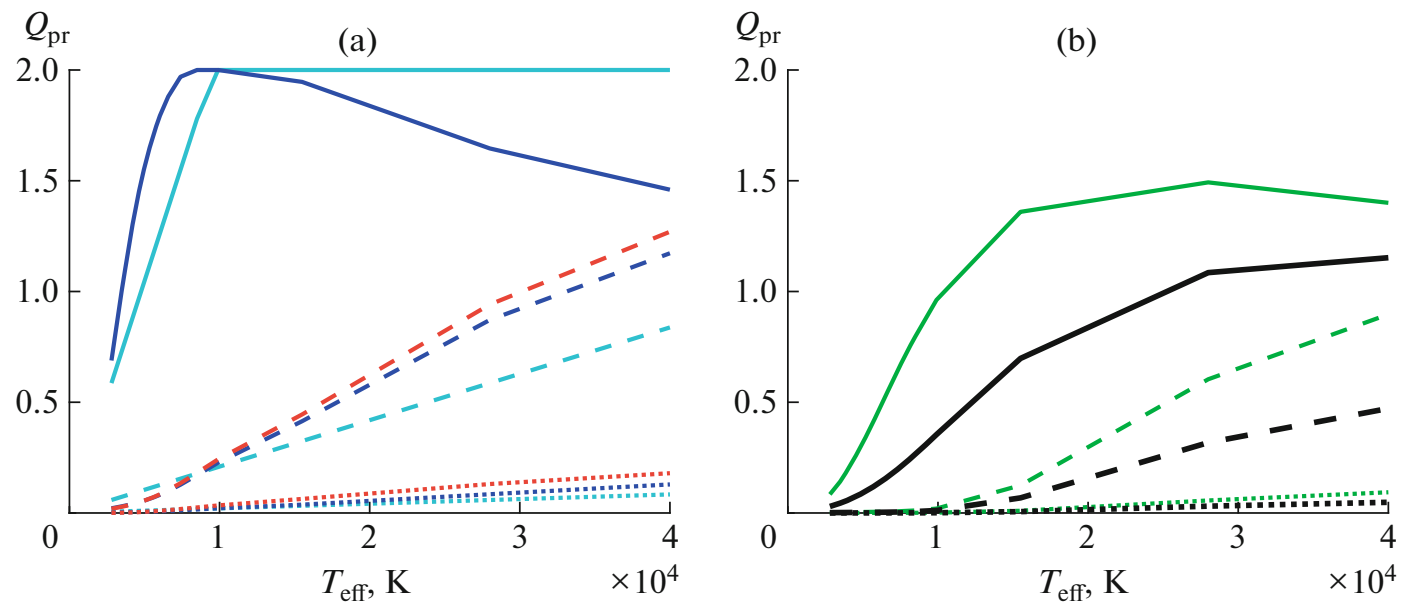

Fig. 2. The radiation pressure efficiency factor $Q_{\mathrm{pr}}$ in dependence on the effective temperature of the radiation source for carbonaceous (a) and silicate (b) dust grains. The designations are the same as those in Fig. 1; in (a), the dotted, dashed, and solid lightblue curves show the temperature dependences of the radiation pressure efficiency factor obtained under the Rayleigh scattering approximation for carbonaceous grains of three sizes-10, 100, and $1000 \AA$ (or $0.1 \mu \mathrm{m}$ ), respectively.

The total force of the radiation pressure is also determined by the shape of the radiation spectrum rather than only the factor $Q_{\mathrm{pr}}(\mathrm{v})$. To illustrate this dependence, we show in Fig. 2 how the value of the radiation pressure efficiency factor changes with the temperature $T$ of the Planck spectrum. It is seen from these diagrams that the values of $Q_{\mathrm{pr}}(T)$ for silicate grains is smaller than those for carbonaceous grains of the same size. In Fig. 2b, the green and black curves present the values of $Q_{\mathrm{pr}}(T)$ for dense grains and grains with a porosity of $50 \%$, respectively. It is also seen here that the smaller is the mean density due to accounting for the porous structure of silicates, the smaller is the value of $Q_{\mathrm{pr}}(T)$ by the same percentage. Thus, again, we have received a confirmation that the accounting for the porosity of silicate dust is apparently of no substantial significance in the context of solving the equation of motion of a grain.

Moreover, in Fig. 2a, the light-blue curves present the temperature dependences of the radiation pressure efficiency factor obtained under the Rayleigh scattering approximation, i.e., $Q_{\mathrm{pr}}=2 \pi a / \lambda$ (this approach was used in a paper [40]). It is seen that this approximate approach results in overestimating the values of $Q_{\mathrm{pr}}$ for large dust grains $(0.1 \mu \mathrm{m}$ in radius $)$ and underestimating it for smaller ones. The consequences of these discrepancies will be discussed below.

For a dust particle of a specified size and composition (density), we may estimate (within constant factor) the ratio of the accelerations induced by the radiation pressure and the gravitational force. This ratio is proportional to the quantity $Q_{\mathrm{pr}} / a / \rho$. In Fig. 3 , the latter is shown in dependence on the effective temperature. We assumed that the density of graphite, PAH, and silicate grains is $2.2,1.5$, and $3.3 \mathrm{~g} / \mathrm{cm}^{3}$, respectively. It is seen that silicate grains should be swept out of the galactic disc least effectively, while the radiation pressure impact on PAH grains is strongest. The values of the considered ratio are small for large graphite grains at high values of $T_{\text {eff }}$; however, when $T_{\text {eff }}$ is low, the values of $Q_{\mathrm{pr}} / a / \rho$ for these grains are higher than those for grains of all other types. This value of the ratio is hardly reached in disc galaxies, but it may occur in the systems, the stellar population of which is older.

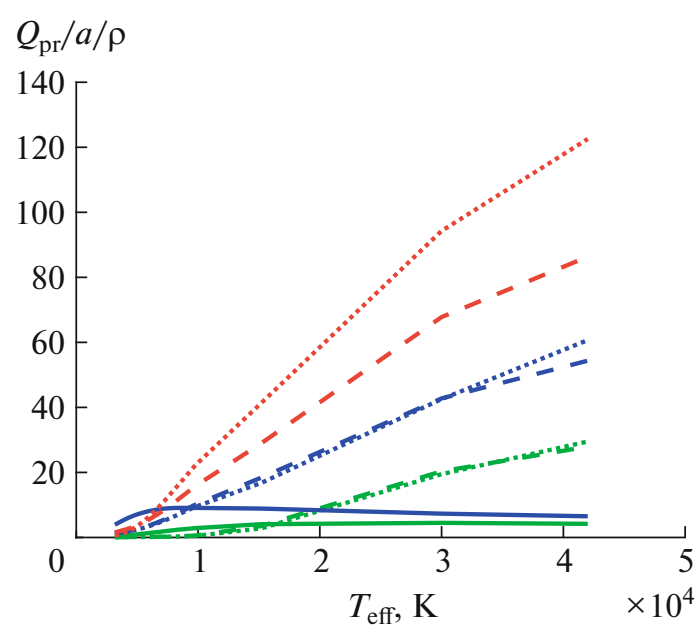

Fig. 3. The ratio $Q_{\mathrm{pr}} / a / \rho$, where $a$ is the particle radius expressed in micrometers and $\rho$ is the density expressed in $\mathrm{g} / \mathrm{cm}^{3}$; the dotted, dashed, and solid lines correspond to sizes of $0.001,0.01$, and $0.1 \mu \mathrm{m}$, respectively. The lines for graphite, $\mathrm{PAH}$, and silicate grains are shown blue, red, and green, respectively. 


\subsection{The Forces Acting on Dust in the Galaxy Vicinity}

In this subsection, we will consider the main factors that determine the dynamics of dust in the Galaxy: the radiation pressure, the gravitation, and the interstellar gas drag. To calculate the radiation pressure and gravitation forces, we used the precise data on the distribution of stars in the Galaxy (see below). The influence of the galactic wind is ignored in the present study. Since we assume that dust grains are electrically neutral, we do not consider the magnetic field impact on the motion of grains (see the discussion in Section 4), though the model has been provided with special structures for this.

2.2.1. The radiation pressure force. The radiation pressure experienced by a dust particle on the part of a point source with the luminosity $L$ at a distance $r$ is

$$
f_{\mathrm{r}}=\frac{L \sigma_{\mathrm{d}}}{4 \pi c r^{2}} Q_{\mathrm{pr}}
$$

where $c$ is the velocity of light and $\sigma_{\mathrm{d}}=\pi a^{2}$ is the cross-section of a dust particle with a radius $a$.

To totally consider the contribution of different stellar populations of the Galaxy to the radiation pressure, we use the luminosity function divided into $m$ groups (in this paper, $m=21$ ), each of which is characterized by the luminosity $L_{i}$, the effective temperature $T_{i}$, and its own parameters of the distribution in the Galaxy. We assume that the volume density $\varphi_{i}$ of stars in the $i$ th group at the point with cylindrical coordinates $(r, z)$ is defined by the following expression

$$
\varphi_{i}(z, r)=\varphi_{0}^{i} \exp \left(-|z| / \beta_{i}\right) \exp \left(-r / r_{\mathrm{h}}\right),
$$

where $\varphi_{0}^{i}$ is the number of stars in the $i$ th group per volume unit in the center of the Galaxy, $\beta_{i}$ is the exponential scale height, and $r_{\mathrm{h}}$ is the radial scale, which is assumed to be $5 \mathrm{kpc}$ for all objects. To group the stars and to select the parameters for each of the groups, the data from a paper [45] were used, and these data were also compared and corrected according to the data in a paper [46]. The luminosity function was normalized in such a way that the mass of stars of the galactic disc was $\sim\left(6 \times 10^{10}\right) M_{\odot}[47]$. In this case, the total luminosity is $\sim\left(2.8 \times 10^{10}\right) L_{\odot}$ in the V band. For control, we compared the obtained luminosity function with more recent results $[48,49]$, but no significant differences were found.

A dust particle at a point with a radius-vector $\vec{r}_{0}$ experiences the radiation pressure force on the part of an elementary volume $d \tau$ at a point with a radius-vector $\vec{r}$ (i.e., at a point with cylindrical coordinates $(r, z)$ )

$$
\begin{gathered}
d \vec{F}_{\mathrm{pr}}=\sum_{i} \frac{L_{i} \sigma_{d}}{4 \pi c} Q_{\mathrm{pr}} \varphi_{i} \\
\times \exp \left(-|z| / \beta_{i}\right) \exp \left(-r / r_{\mathrm{h}}\right) \frac{\vec{r}_{0}-\vec{r}}{\left|\vec{r}_{0}-\vec{r}\right|^{3}} d \tau .
\end{gathered}
$$

By integrating this expression over the entire volume of the Galaxy, we derive the formula for the radiation pressure force acting on a dust particle

$$
\begin{aligned}
\vec{F}_{\mathrm{r}}= & \int_{\tau} \sum_{i} \frac{L_{i} \sigma_{d}}{4 \pi c} Q_{\mathrm{pr}} \varphi_{i} \exp \left(-|z| / \beta_{i}\right) \\
& \times \exp \left(-r / r_{\mathrm{h}}\right) \frac{\vec{r}_{0}-\vec{r}}{\left|\vec{r}_{0}-\vec{r}\right|^{3}} d \tau .
\end{aligned}
$$

2.2.2. The gravitational attraction force. A dust particle at a point with a radius-vector $\vec{r}_{0}$ experiences the attraction force from an elementary volume $d \tau$ at a point with a radius-vector $\vec{r}$

$$
\begin{aligned}
d \vec{F}_{\mathrm{g}} & =\sum_{i} G M_{i} m_{\mathrm{d}} \varphi_{i} \exp \left(-|z| / \beta_{i}\right) \\
& \times \exp \left(-r / r_{\mathrm{h}}\right) \frac{\vec{r}_{0}-\vec{r}}{\left|\vec{r}_{0}-\vec{r}\right|^{3}} d \tau .
\end{aligned}
$$

By integrating this expression over the entire volume, we derive the formula for the attraction force of the Galaxy acting on a dust particle

$$
\begin{aligned}
\vec{F}_{\mathrm{g}} & =\int_{\tau} G M_{i} m_{\mathrm{d}} \varphi_{i} \exp \left(-|z| / \beta_{i}\right) \\
& \times \exp \left(-r / r_{\mathrm{h}}\right) \frac{\vec{r}_{0}-\vec{r}}{\left|\vec{r}_{0}-\vec{r}\right|^{3}} d \tau,
\end{aligned}
$$

where $G$ is the gravitational force and $m_{\mathrm{d}}$ is the mass of a dust particle.

Moreover, we separately considered the influence of the dark matter halo. The halo mass within a radius $R$ was calculated from the Navarro-Frenk-White profile [50] of the dark matter density [51]

$$
M(R)=4 \pi \rho_{c} \delta_{0} R_{s}^{3}\left[\ln \left(1+R / R_{s}\right)-\frac{R / R_{s}}{1+R / R_{s}}\right],
$$

where

$$
\begin{gathered}
\delta_{0}=\frac{200}{3} \frac{C^{3}}{\ln (1+C)-C /(1+C)}, \\
R=\sqrt{r^{2}+z^{2}} .
\end{gathered}
$$

It was assumed that the parameter $C=10$ and the scaling parameter $R_{\mathrm{s}}=16 \mathrm{kpc}$ [52]. To calculate the critical density, the value of the dimensionless Hubble constant was assumed to be $h=H /(100(\mathrm{~km} / \mathrm{s}) / \mathrm{Mpc})=0.67$.

From these assumptions, we calculated the components of the force acting on a dust particle along the galactocentric radius and the altitude on the part of the dark halo

$$
F_{\text {halo }, r}=-\frac{r G m_{\mathrm{d}} M(R)}{\left(r^{2}+z^{2}\right)^{3 / 2}}, \quad F_{\text {halo }, z}=-\frac{z G m_{\mathrm{d}} M(R)}{\left(r^{2}+z^{2}\right)^{3 / 2}} .
$$

The total mass distribution of the baryonic and dark matter in the Galaxy was used to calculate the azimuthal component of the rotation curve $V_{\mathrm{R}}(r, z)$. 
2.2.3. The drag force of interstellar gas. Even at the gas density values corresponding to the intergalactic space, the dragging of dust particles by surrounding gas plays an important role. Both the direct and Coulomb collisions with ions and electrons contribute to the drag force. This process was considered at length in a paper [53]. When moving through partially ionized hydrogen, a particle with a charge $z_{\mathrm{d}}$ experiences the drag force

$$
F_{\text {drag }}=2 \pi a^{2} k \operatorname{Tn}\left[G_{0}(s)+\varphi^{2} \ln (\Lambda) G_{2}(s)\right],
$$

where

$$
\begin{gathered}
s=\left[\frac{m_{\mathrm{H}} V^{2}}{2 k T}\right]^{1 / 2}, \\
G_{0}(s) \approx \frac{8 s}{3 \sqrt{\pi}}\left[1+\frac{9 \pi}{64} s^{2}\right]^{1 / 2}, \\
\varphi=\frac{z_{g} e^{2}}{a k T}, \\
\Lambda=\frac{3}{2 a e \varphi}\left[\frac{k T}{\pi n_{\mathrm{e}}}\right]^{1 / 2},
\end{gathered}
$$

where $n$ is the hydrogen concentration, $e$ is the electron charge, and $n_{\mathrm{e}}$ is the concentration of electrons. Though the Coulomb drag is considered in the general formulation of the model, we ignore the particle's charge in this analysis.

To estimate the density distribution of the gas, through which the particles is moving, we used the data from a paper [54]:

$$
n(r, z)=n_{0} \exp \left(-r / r_{\mathrm{n}}\right) \exp \left(-z / z_{\mathrm{n}}\right),
$$

where $n_{0}=0.9 \mathrm{~cm}^{-3}, r_{\mathrm{n}}=3150 \mathrm{pc}$. The vertical scale height is expressed as

$$
z_{\mathrm{n}}=h_{0} \exp \left(\left(r-R_{\odot}\right) / r_{\mathrm{n}}\right),
$$

where $h_{0}=150 \mathrm{pc}$. The gas rotates around the symmetry axis of the Galaxy with a constant velocity $V_{\text {gas }}^{\varphi}=V_{\mathrm{R}}(r, z)$. The components of the gas velocity $V_{\text {gas }}^{r}$ and $V_{\text {gas }}^{z}$ are always zero.

\subsection{The Particle's Equation of Motion}

With accounting for the above-considered forces, the particle's equation of motion is written in the following way:

$$
\frac{d \vec{v}}{d t}=\vec{F}_{\mathrm{r}}+\vec{F}_{\mathrm{g}}-F_{\mathrm{drag}} \frac{\vec{V}}{V}+\vec{F}_{\text {halo }},
$$

where $v$ is the velocity of a dust particle. To estimate whether dust particles can leave the disc and pass into the halo for the lifetime of the Galaxy, we integrated the equation of motion up to $10 \mathrm{Gyr}$. The particles start to move from different initial coordinates; and they are considered as having left the galactic disc, if they reached altitude of $200 \mathrm{pc}$ for $10 \mathrm{Gyr}$. To account for the rotation of the Galaxy, the equation of motion was integrated in the three-dimensional cylindrical coordinate system, though all of the forces considered in the present analysis exhibit no azimuthal dependence. The evolution of the Galaxy's parameters with time was ignored.

At the initial time moment, the components of the particle velocity $v_{r}$ and $v_{z}$ were set equal to zero. The velocity component $v_{\varphi}$ was specified according to the rotation curve $V_{\mathrm{R}}(r, z)$. All three velocity components of a particle may vary in the course of calculations.

\subsection{Sources of Dust}

The main sources of dust in the Galaxy are stars at late evolution phases and envelopes of supernovae (see [55] and the references therein). They produce $\sim 90 \%$ of the whole dust in the Galaxy. The main components of dust produced by these sources are graphite and silicate grains [56]. According to the data of a paper [57], small grains, including PAHs, compose the main portion of the whole mass of interstellar dust.

In this study, the information on the dust production is the main source of uncertainty. According to the data by different authors, the mass loss rates of stars of different types diverge by more than an order of magnitude $[58,59]$. The contribution of each type of the sources to the dust production is estimated also ambiguously [60]. Up to now, the probable contribution of molecular clouds to the total balance of the dust production have been under discussion [61]; however, when estimating the rate of the dust sweeping out of the Galaxy, the dust that is present (formed) in molecular clouds may be ignored in any case. The estimates show that, if the concentration of the matter in dark cold clouds exceeds $10 \mathrm{~cm}^{-3}$, the dust is frozen into a cloud and does not participate in the large-scale motion connected with the stellar radiation pressure [62]. Consequently, in this analysis, the scale height for the main sources of dust was assumed to be the scale height for the stars that have already evolved, which approximately amounts to $350 \mathrm{pc}[63,64]$.

\section{CALCULATION RESULTS}

The main purpose of this paper is to study the dynamic evolution of graphite and silicate grains and to estimate the general rate of the dust loss from a galactic disc. We considered three representative values of the grain radius $-0.001,0.01$, and $0.1 \mu \mathrm{m}-$ for PAHs and very small grains (VSG), medium-size grains, and "classical" dust grains, respectively. A portion of dust grains of each of the sizes was calculated in accordance with the distribution [57]. In the frames of this model, fine dust (including PAHs as an intermediate member between grains and macromolecules) 


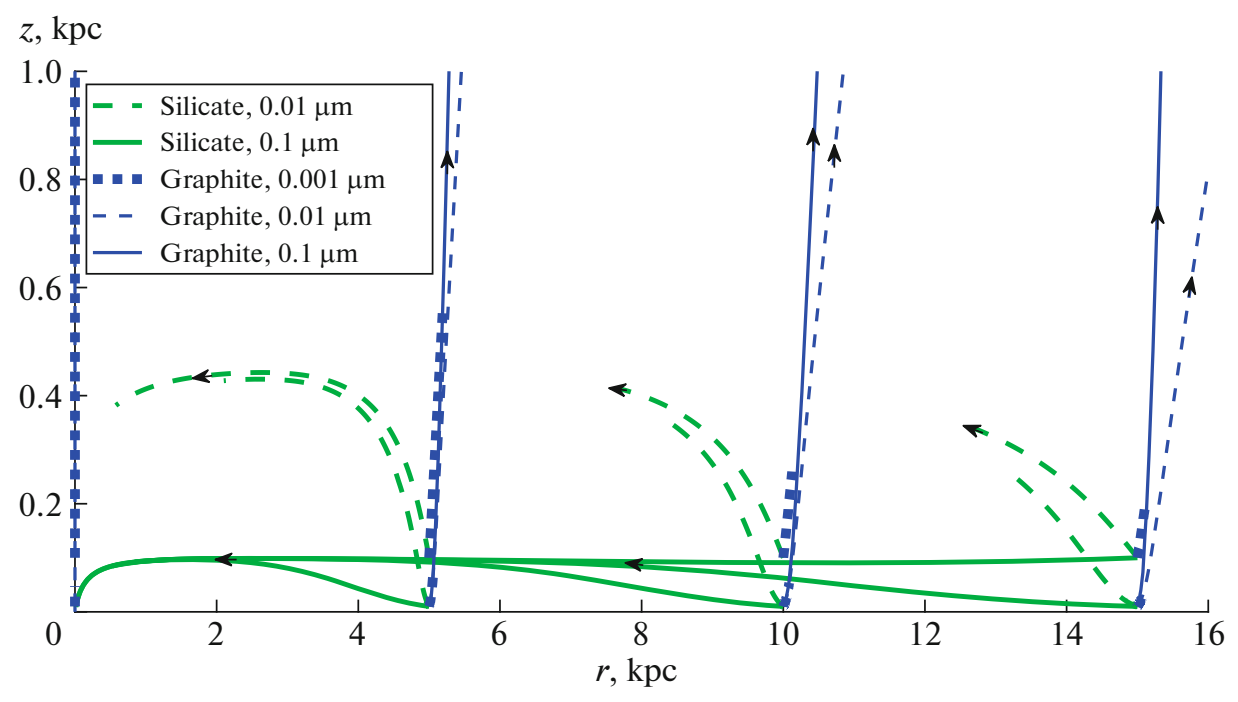

Fig. 4. The trajectories of grains obtained under ignoring the Galactic rotation: the dotted, dashed, and solid lines correspond to sizes of $0.001,0.01$, and $0.1 \mu \mathrm{m}$, respectively. The blue and green lines present the trajectories of graphite and dense silicate grains, respectively.

amounts approximately $80 \%$ of the total dust mass, while the portions of medium- and large-size grains are roughly $10 \%$ each. From these suppositions, we believe that it is fine grains, the motion of which should necessarily be traced, since they are to make the main contribution to the sweeping of solid matter out of a galactic disc. For these grains, we considered two cases of the optical characteristics-for PAH and graphite. As will be shown below, the choice of a particular type of grains does not influence much the estimates of the dust mass loss.

\subsection{Calculation Results Ignoring the Galactic Rotation}

In this subsection, to demonstrate the differences in the motion of grains with different characteristics (dense and porous silicate grains and carbonaceous grains with the properties of graphite and PAH), we present the calculation results performed for the case when the rotation was not accounted for. In Fig. 4, we show the trajectories of dense silicate and graphite grains for different initial positions on the galactocentric radius $(0,5,10$, and $15 \mathrm{kpc})$ and two initial altitudes ( 10 and $100 \mathrm{pc}$ ). It is seen that the grains of different chemical composition move in a different way. As can be expected from the results shown in Fig. 3, silicate grains (green lines) only insignificantly change their positions on the $z$ axis. The grains of medium sizes (dashed lines) rise most noticeably above the disc plane. Small silicate particles cover such short distances that their motion cannot be traced in the diagram of Fig. 4, since their ratio $Q_{\mathrm{pr}} / a / \rho$ in the stars of most types turns out to be smaller than that for medium-size grains.
Medium and large graphite grains (dashed and solid blue lines), on the opposite, rise to a significant altitude regardless of the initial position. The exception is large grains being in the center of the Galaxy below $z=10 \mathrm{pc}$; these grains move toward the disc plane ("downward"). Small graphite grains are effectively swept out of the Galaxy near its center. The farther are their positions from the center, the lower are their effectiveness.

In Fig. 5, the trajectories of dense and porous silicate grains are compared. It is seen that dense grains move somewhat faster than porous ones; however, no qualitative differences in the behavior of their motion are observed. This confirms the previous conclusion that the accounting for the probable porosity of silicate grains does not play any significant role in estimating the general rate of the dust sweeping out of the Galaxy.

For considering the motion of carbonaceous particles, the choice of optical properties turns out to be more important. In Fig. 6, the trajectories of carbonaceous grains are shown for the same initial altitude $(100 \mathrm{pc})$, different initial positions on the radius $(0,5$, 10 , and $15 \mathrm{kpc}$ ), and different optical properties (graphite and PAH). Since the grains of different types differ in density (the density of PAH grains is lower than that of graphite ones, while the latter is, in turn, lower than the density of silicate grains), the grains of the same size but different chemical composition experience different gravitational impacts; and, consequently, their trajectories noticeably diverge. Medium-size grains with the optical properties of graphite, when rising above the galactic disc, noticeably shift to the symmetry axis of the Galaxy (Fig. 6a). Since the gravitational force is proportional to the grain mass, large particles experience even a stronger 


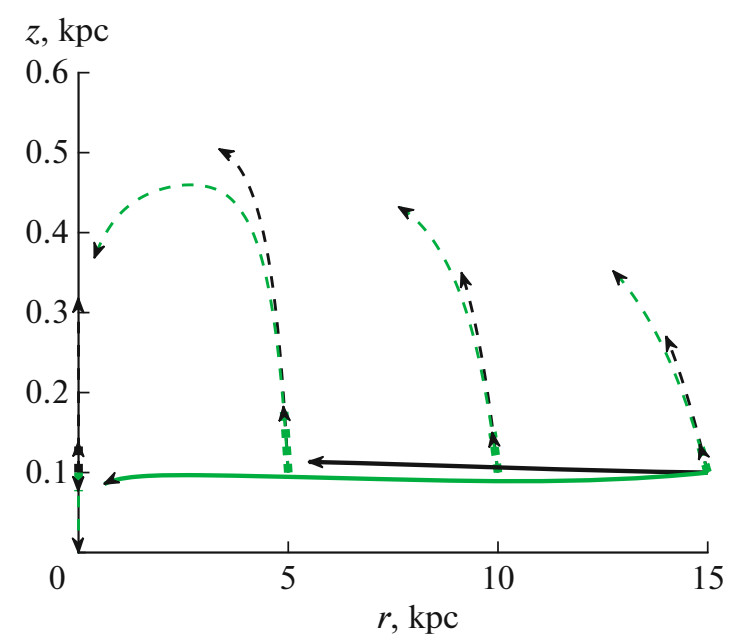

Fig. 5. Examples of the trajectories of silicate grains: the dotted, dashed, and solid lines correspond to sizes of 0.001 , 0.01 , and $0.1 \mu \mathrm{m}$, respectively. The trajectories of dense and porous grains are shown green and black, respectively.

influence of the baryon and dark matter. For large graphite grains, there is a limiting value of the altitude $z$, which they can reach. Small graphite particles cover a substantially shorter distance than large ones for the same time, i.e., they are swept out much less efficiently. Because of this, their trajectories are almost indistinguishable in the diagram.

In Fig. 6b, the trajectories of small and medium particles with the optical properties of PAH are presented. Their motion essentially differs from that of graphite grains. Small and medium PAH grains, being at larger distances from the Galaxy center, move away from the center along inclined trajectories, even if the rotation is ignored, i.e., the motion along the Galactic disc increasingly dominates the vertical motion. This

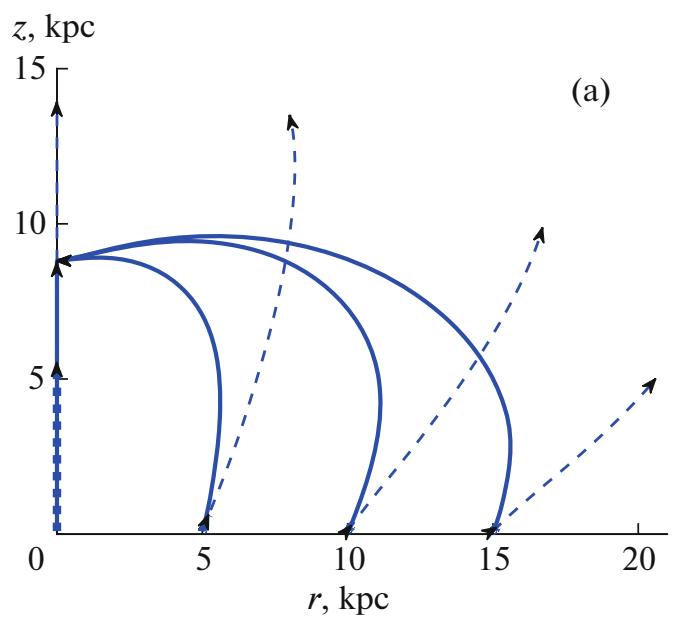

tendency becomes stronger, if the rotation is considered (see below).

The importance of the correct accounting for the optical properties of grains can also be illustrated by comparing the trajectories of carbonaceous grains obtained with the use of two different approaches to determining $Q_{\mathrm{pr}}$ : (i) calculations by formula (1) with the subsequent averaging over the Planck spectral distribution of the energy for the assumed temperatures of radiation sources $T_{\text {eff }}$ and (ii) calculations according to the simplified formula $Q_{\mathrm{pr}}=2 \pi a /\left(0.3 / T_{\text {eff }}\right)$. In Fig. 7, we show how the vertical position of a grain changes with time according to different methods for determining $Q_{\mathrm{pr}}$, if the grain initially was at a zero point on the galactocentric radius. The simplified case is shown with light-blue curves. It is seen that the use of different values of the radiation pressure efficiency factor noticeably changes the sweeping-out pattern for small particles, which are of particular interest, since they amount a substantial portion of the interstellar dust. In simulations with the values of $Q_{\mathrm{pr}}$ that are physically grounded better (calculated with formula (1)), small grains, as is presented in Fig. 7a, may reach the altitudes twice as high as those obtained with the Rayleigh approximation. Moreover, Fig. 7d shows that the use of the Rayleigh approximation in calculations of the radiation pressure efficiency factor for large particles yields, on the contrary, an overestimated value of the altitude that can be reached above the disc plane.

\subsection{Calculation Results Accounting for the Galactic Rotation}

If the rotation is considered in the initial velocity of a dust particle, all of the particles regardless of their initial galactocentric coordinates move toward the

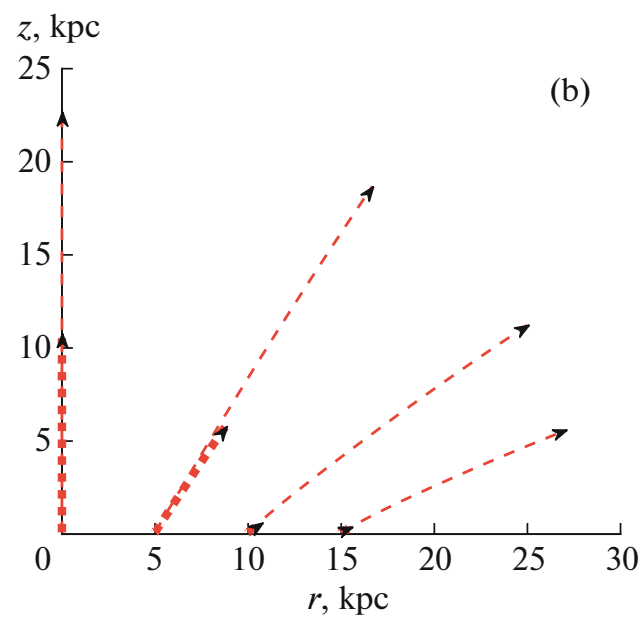

Fig. 6. Examples of the trajectories of graphite grains (a) and PAHs (b); the dotted, dashed, and solid lines correspond to sizes of $0.001,0.01$, and $0.1 \mu \mathrm{m}$, respectively. The blue and red lines present the trajectories of graphite grains and PAHs, respectively. 

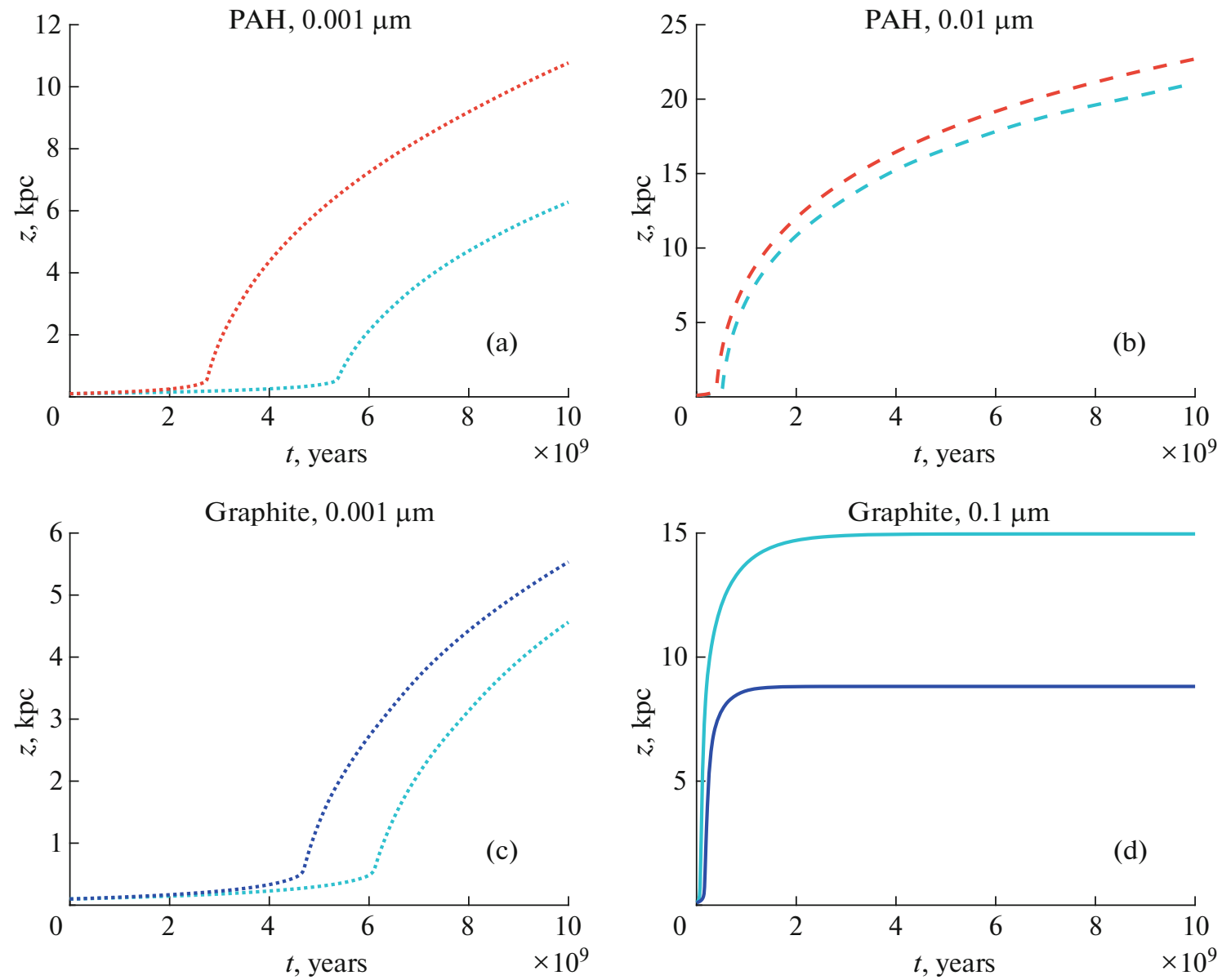

Fig. 7. The time dependence of the altitude above the disc plane for PAHs (a, b) and graphite grains (c, d) at $r=0$. The designations are the same as those in Fig. 1; the analogous dependences obtained with the Rayleigh approximation are shown light-blue.

periphery of the Galaxy (except the grains at the symmetry axis of the Galaxy, where $r=0$ ). In Fig. 8, the trajectories of grains, which were calculated with accounting for the rotation, are presented for several initial positions on the galactocentric radius $(0,5,10$, and $15 \mathrm{kpc}$ ) and two initial altitudes (10 and $100 \mathrm{pc}$ ).

The comparison of Figs. 4 and 8 shows that the character of the graphite grain motion (blue lines) does not essentially change after accounting for the rotation. For silicate grains (green lines), the initial rotation turns out to be of key importance: they move in the opposite direction, away from the center of the Galaxy, if the rotation is included into the model. However, in a vertical direction, silicate grains move almost identically whether the rotation was taken into account or ignored.

Figure 9 separately presents examples of the trajectories of carbonaceous grains with the optical properties of graphite and PAH; their sizes are 0.001, 0.01, and $0.1 \mu \mathrm{m}$ (the latter is only for graphite grains). Various initial positions of grains on the galactocentric radius $(0,5,10$, and $15 \mathrm{kpc})$ are considered for an initial altitude of $100 \mathrm{pc}$. It is seen from the diagrams that, if the initial radius is not zero, the grains with the optical properties of graphite reach the highest position (the closer to the center of Galaxy is the initial point, the higher is this altitude) and start to move downward. The grains with the optical properties of PAH move also gradually slower in the vertical direction, though they do not reach the maximal altitude at $r<40 \mathrm{kpc}$. In both cases, the motion along the Galactic disc strongly predominates over the vertical motion. This peculiarity may explain the smoothing of the metallicity gradients $[65,66]$, which is observed at galactocentric distances larger than $10 \mathrm{kpc}$. In this case, small particles also move with essentially lower velocities, i.e., they are swept out less effectively.

In Fig. 10, the analogous trajectories of silicate grains are shown. Large and small particles do not substantially change their positions in altitude, and only medium-size grains move noticeably. These results suggest that silicate dust does not leave the Galaxy due to the considered mechanism, i.e., the stellar radiation pressure effect; however, this dust may be swept out to large distances in the radial direction. Nevertheless, it is worth noting that, in the interstellar 


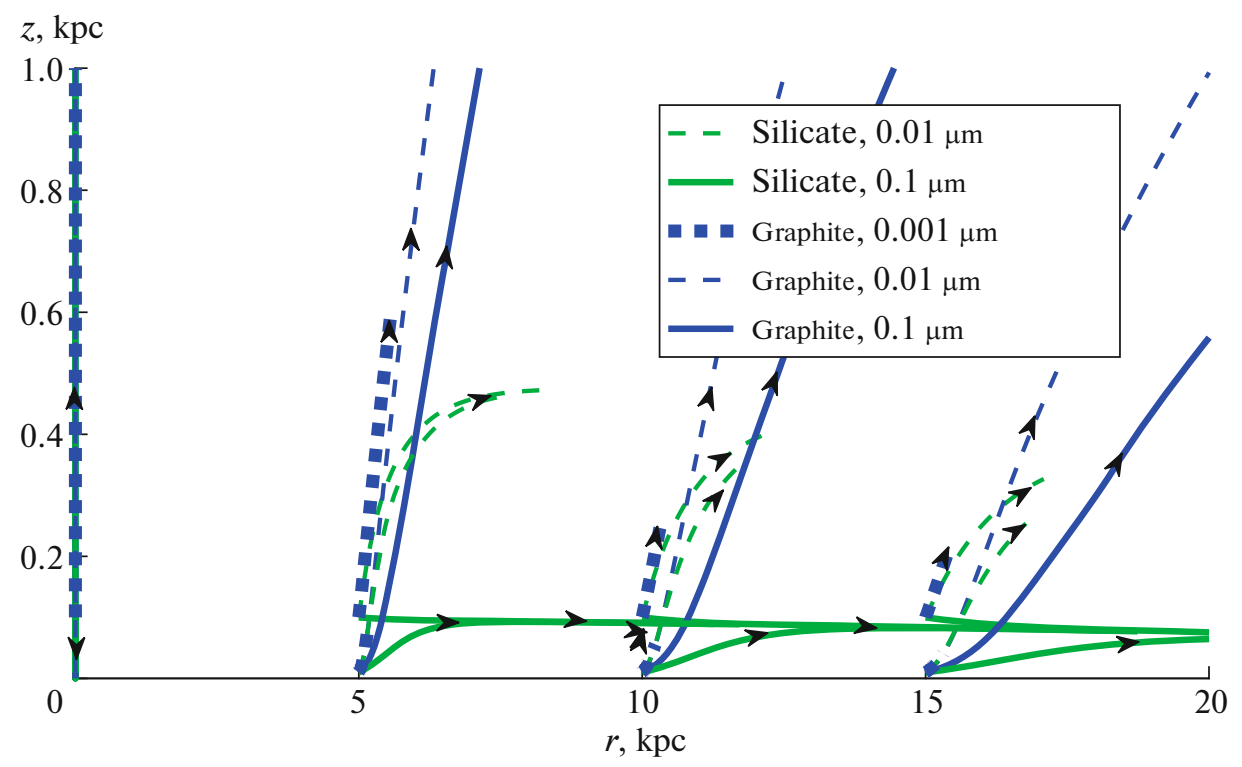

Fig. 8. The trajectories of grains: the dotted, dashed, and solid lines correspond to sizes of $0.001,0.01$, and $0.1 \mu \mathrm{m}$, respectively. The blue and green lines present the trajectories of graphite and silicate grains, respectively.
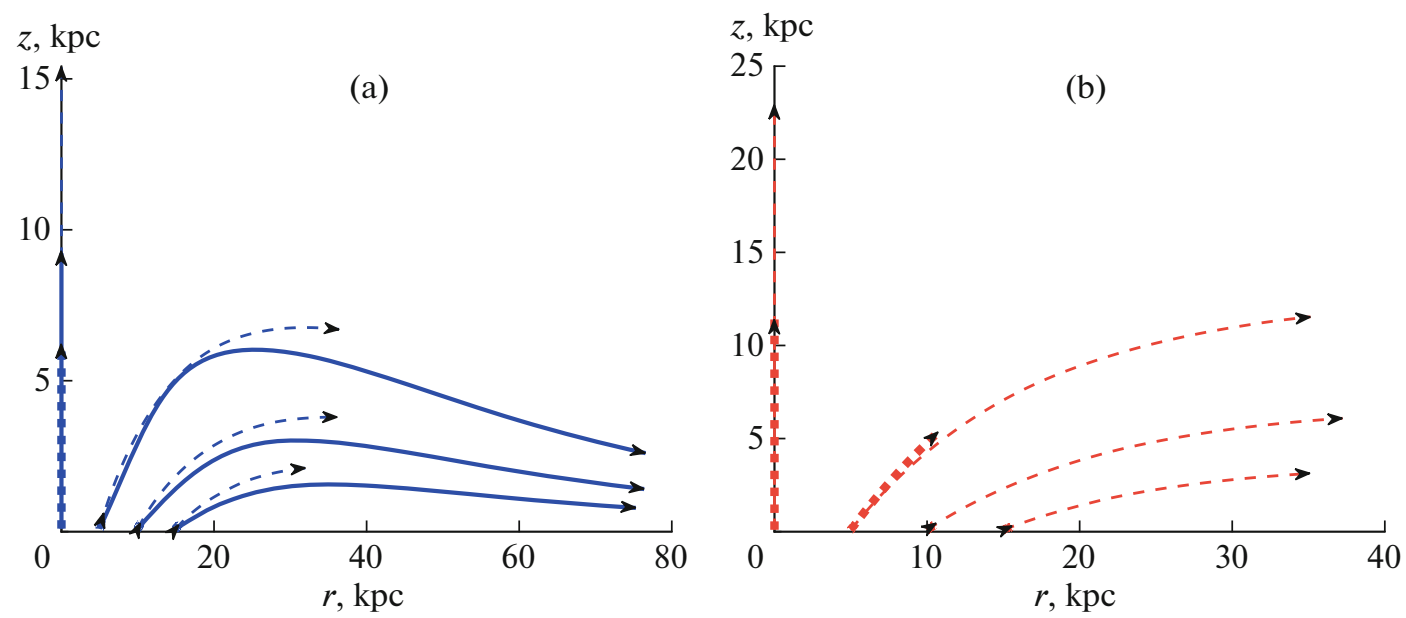

Fig. 9. Examples of the trajectories of graphite grains (a) and PAHs (b): the dotted, dashed, and solid lines correspond to sizes of $0.001,0.01$, and $0.1 \mu \mathrm{m}$, respectively. The blue and red lines present the trajectories of graphite grains and PAHs, respectively.

space, silicate particles are most likely covered with carbon mantles [57] and their optical properties differ from those of purely silicate particles. However, a reliable model for the sweeping-out of grains of this kind by the stellar radiation pressure should also contain the processes of formation and destruction of mantles, which is beyond a scope of the present analysis.

\subsection{The Rate of Sweeping Grains out of the Galaxy}

In general, as is seen from Figs. 8-10, not every particle can leave the Galaxy. This capability depends on several factors: the estimated time, as well as the initial position, the chemical composition, and the size of a grain. In this study, we are focused on the conditions, under which dust particles leave a thin disc of the Galaxy for the estimated time (10 Gyr). Thus, the task is to determine the limiting altitude $z_{\text {lim }}(r)$, beginning from which all the dust of a certain type will certainly leave the Galactic disc.

The dependences of $z_{\lim }(r)$ on the initial position of a grain on the galactocentric radius for three chosen sizes of grains are shown in Fig. 11. All of the dust grains, which were formed above the boundary shown 


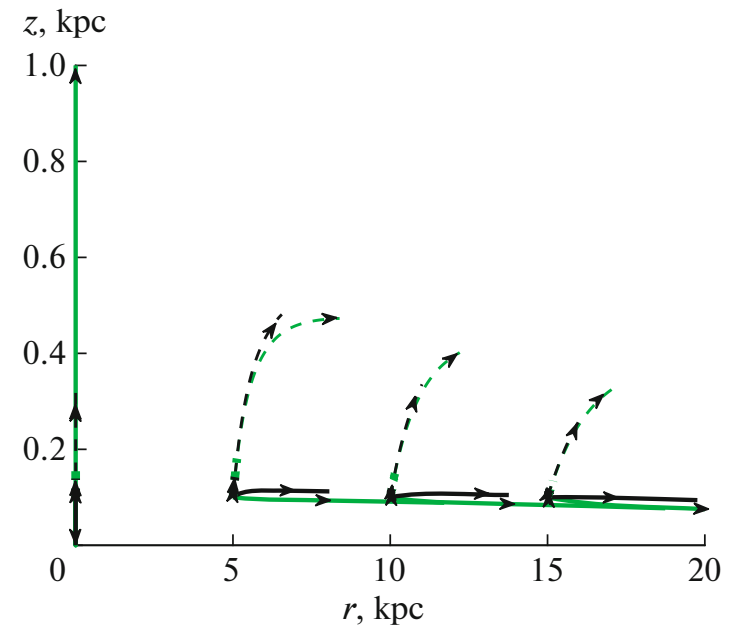

Fig. 10. Examples of the trajectories of silicate grains: the dotted, dashed, and solid lines correspond to sizes of $0.001,0.01$, and $0.1 \mu \mathrm{m}$, respectively. The green and black lines present the trajectories of dense and porous grains, respectively.

in the diagram, leave the Galactic disc. It is seen that grains of medium and large sizes are swept out of the Galaxy most effectively: for the estimated time, almost the entire Galactic disc has become free of large dust grains all large dust grains leave the Galactic disc; the exception is a narrow area near the disc plane as well as the central part. However, since small grains form a large portion of the interstellar dust bulk [57], it is these particles that mostly contribute to the mass loss due to the considered processes. In this case, the limiting altitude becomes substantially dependent on the optical properties of a grain. In Fig. 11, the curves calculated for grains with the optical properties of graphite and PAH are shown blue and red, respectively. Small PAH grains, which are in the central region of the disc, leave it if they were formed above a few parsecs; at the same time, on the disc periphery, a small PAH grain may escape from the disc, only if it was formed at the altitude of about $50 \mathrm{pc}$ and higher. For small graphite particles, the limiting altitude at the disc periphery is approximately $100 \mathrm{pc}$.

From the distribution of dust sources in the disc, the surface density of the loss rate of the dust mass can be calculated. Since, according to Fig. 11, dust is swept out of the central part of the Galaxy more effectively than out of the periphery, we should expect that the larger mass is lost from the center and this value decreases with moving away from the center along the galactic radius. Figure 12 presents the surface density of the mass loss rate in dependence on the galactocentric distance for three chosen sizes of grains. The main contribution to the dust loss is made by small particles, which add up to a larger portion of the total mass of the interstellar dust in the assumed model. We note that, as distinct from the limiting altitude, the summed loss

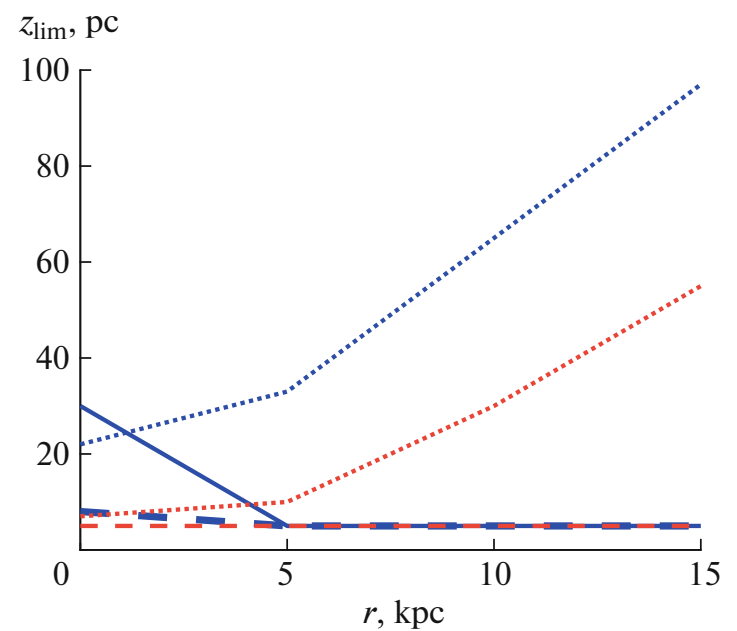

Fig. 11. The altitude, beginning from which dust leave the Galactic disk for the estimated time, versus the initial coordinate on the galactic radius; the dotted, dashed, and solid lines correspond to sizes of $0.001,0.01$, and $0.1 \mu \mathrm{m}$, respectively. The blue and red lines present the limiting altitude for graphite grains and PAHs, respectively.

rate of small particles from a unit surface is almost independent of the assumption on their optical properties, since almost all dust grains are formed above the limiting values of $z$ obtained here (Fig. 11).

When describing probable causes of the development of the radial gradient of metallicity in disc galaxies (more specifically, in the Milky Way), our concern is also with the surface density of the dust mass loss rate normalized on the surface density of gas, since the abundance of metals in the Galaxy is also a relative quantity. This quantity is shown in Fig. 13. It is seen from this diagram that the absolute value of the dust mass loss actually decreases toward the periphery of the Galaxy, but its relative contribution to the formation of the gas metallicity, on the contrary, somewhat grows with moving away from the center. This means that the sweeping-out of dust by the stellar radiation pressure may contribute to the formation of the radial gradient of heavy elements contained in the dust.

The integration over all sizes of grains and the entire volume of the Galaxy yields $0.03 M_{\odot}$ per year for the general rate of the mass loss in the form of dust. The obtained value is comparable to that of heavy elements ejected by the wind, which suggests that the described mechanism of sweeping dust out of the Galaxy is a significant factor in the processes of the matter interchange with the intergalactic medium and may explain the presence of circumgalactic dust structures.

\section{DISCUSSION OF THE RESULTS}

As has been noted in the Introduction, dust is swept out of a galactic disc not only in the Milky Way, but generally in most disc galaxies, due to which dust 


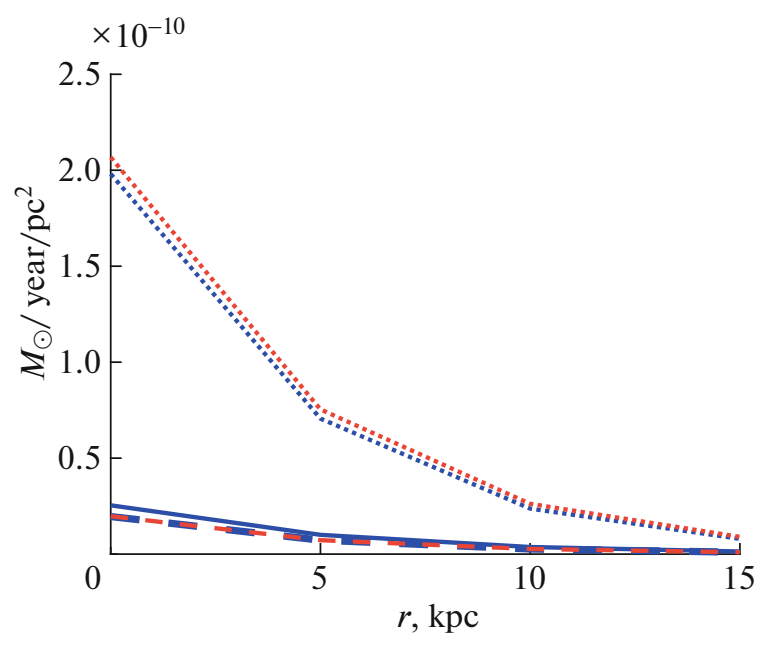

Fig. 12. The surface density of the mass loss rate in dependence on the galactocentric distance. The dotted, dashed, and solid lines correspond to sizes of $0.001,0.01$, and $0.1 \mu \mathrm{m}$, respectively.

becomes a significant component of the circumgalactic and intergalactic medium. According to our calculations and other authors, the stellar radiation pressure is an important factor that determines the effectiveness of this process. The sweeping-out of dust by the stellar radiation pressure were earlier considered by Shustov and Vibe (see [40]); however, the currently available new data on the properties of dust in galactic discs and the evolution of dust in the circumgalactic space make us revert to this issue. As distinct from [40], the present model has been improved in the following way.

-The values of the factor $Q_{\mathrm{pr}}$ for grains of different chemical composition and structure were specified more accurately. It has been shown that a choice of the optical properties does not influence much the estimate of the dust loss from a disc, but noticeably changes the trajectories of particles at high galactic altitudes.

-The model has been advanced to the threedimensional formulation of the problem, which allows us to use it in the analysis of more complex motions of dust, for example, with consideration of the magnetic field.

- The model now contains a dark halo, the accounting for which substantially influences the motion of dust at high altitudes.

These improvements resulted in no significant changes in the general behavior of the motion of particles and the dust mass loss rate as compared to those in [40]; however, some model trajectories noticeably differ from those obtained before.

Since the previous and new results agree on the dust loss rate of the Galaxy, we may make some notes about a role of the sweeping-out of dust in the forma-

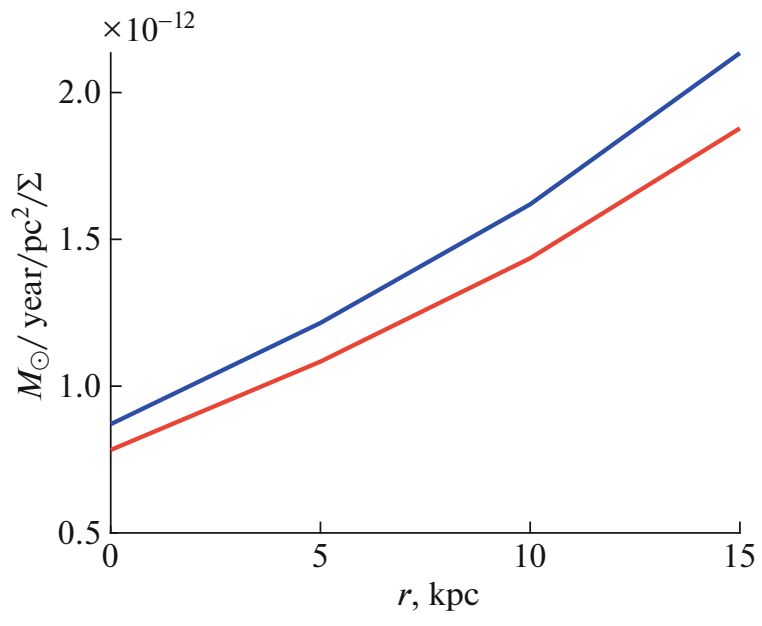

Fig. 13. The surface density of the mass loss rate normalized on the surface density of gas in dependence on the galactocentric distance.

tion of the chemical composition distribution over the Galactic disk. A well-known peculiar feature of this distribution is the metallicity gradients for various chemical elements ( $\mathrm{C}, \mathrm{N}, \mathrm{O}, \mathrm{Ne}, \mathrm{S}, \mathrm{Fe}, \mathrm{Ar}$, and $\mathrm{Al}$ ) (e.g., [67]) observed in disc galaxies. These elements are among the constituents of dust grains [68]. Apparently, if we consider that the sweeping-out of dust, which is caused by the stellar radiation pressure, contributes to the formation of the radial gradients of metallicity, the composition gradients for the elements composing the dust should differ from those absent in the dust. Thus, dust grains contain these elements in different proportions. In addition to the sweeping-out of dust by the radiation pressure, the galactic wind is apparently not the least in the formation of the gradient, but we did not model its contribution here. This factor may be important for dwarf galaxies at $z \sim 2$, where the radial gradients of metallicity are inverted. This suggests that a main portion of the stellar nucleosynthesis products is transported to the galactic periphery by the strong galactic wind induced by the central starburst [69].

For our Galaxy, the comparison of the gradients for different elements yields controversial results. It can be observed that the gradients strongly diverge for such elements like $\mathrm{He}, \mathrm{Ar}$, and $\mathrm{Ne}$ [70], but they are similar for the elements composing dust grains [70, 71]. On the other hand, there are data that evidence the gradient similarity for most elements regardless of their presence in the interstellar dust composition [72]. However, the observational data on the gradient substantially vary; and not always a definite conclusion on the radial dependence of a specified element may be formulated. Presently, the observational data and the model results can be compared only qualitatively. Our results show that the dust sweeping and the depen- 
dence of the sweeping rate on the galactocentric distance should not be ignored.

Interestingly, in most disc galaxies including the Milky Way, it is observed that the dependence of the abundance of heavy elements on the galactocentric distance changes in shape, which manifests itself in decreasing the slope of the radial gradient of metallicity $[65,66]$. At distances of $\sim 10 \mathrm{kpc}$ from the center, the dependence becomes gentler, i.e., the gradients are smoothed. As has been noted above, this may be connected with decreasing the slope of trajectories of grains relative to the Galactic disk with moving away from the Galactic center.

In this paper, we restricted ourselves with the issues connected with the motion of grains at low galactic altitudes. The analysis of the other problems - the dust ejection to large distances, the destruction of dust in the circumstellar space, etc.-will require further improvements in the model. In the present study, we assumed the grains to be electrically neutral and, consequently, ignored the contribution of the magnetic field into their dynamics. However, the grains may be actually charged and, as a result, move along the magnetic field lines of the Galaxy. On the one hand, the magnetic-field strength is insignificant at high galactic altitudes [73], but it contains a substantial irregular component; consequently, the magnetic field will rather cause the dust motion to become chaotic and produce no substantial effect on its global characteristics. Since we are mainly focused here on the general rate of the dust sweeping at different galactocentric radii rather than the exact dynamics of dust grains, we ignored the Lorentz force in calculations of the motion of a grain. On the other hand, the incorporation of the dust destruction processes into the model may enhance the importance of the magnetic field impact. We will analyze the influence of the magnetic field separately in future.

The model can be supplemented with the comprehensive simulations of the destruction processes of dust grains. This process was approximately considered in [40]; however, to date, we have developed a much more thorough model (called SHIVA) for the evolution of dust particles of different sizes [74]. This model will make it possible to understand whether there is a link between the swept dust grains and the appearance of extraplanar PAH particles in our and other galaxies. Since the stellar disc of the Galaxy was most likely formed in relatively early times [75] and the majority of stars-sources of dust-were formed for $\sim 1.5 \mathrm{Gyr}$, there is probably less need to account for evolutionary processes in the context of the formulated problem. Different studies show that there have been no substantial variations in the star formation rate in the Galactic disc for the recent 10 Gyr [76, 77]. Nevertheless, we also plan to apply the model to the other galaxies, including the galaxies with a starburst, for which the galactic wind impact will be included into the model. To consider systems of this kind, one should consider the time-dependent parameters of a galaxy.

Finally, we will note that the patterns of the dust motion considered here for a nonrotating galaxy may indirectly be applied to ecliptic galaxies and suggest that extensive dust structures may also be formed in the vicinity of such systems. In elliptic galaxies, such sources of dust as evolved stars are present. The substantially lower density of the interstellar matter will make it easier to sweep dust out by the stellar radiation pressure. However, as our calculations show (see Fig. 2), the effectiveness of the radiation pressure significantly decreases with lowering the characteristic temperature of stellar populations. This problem deserves special consideration.

\section{CONCLUSIONS}

We analyzed the dynamical evolution of carbonaceous and silicate grains of three radii-0.001, 0.01 , and $0.1 \mu \mathrm{m}$. The resulting force acting on these dust grains in the vicinity of a galaxy was calculated, and the characteristic trajectories were presented. We considered carbonaceous grains (with the optical properties of graphite and $\mathrm{PAH}$ ) and silicate grains (with accounting for the probable porosity).

The calculations have shown that carbonaceous medium-size grains, which add up to a small portion of the interstellar dust bulk, are swept out most effectively. Silicate grains experience a less impact of the radiation pressure and, consequently, do not reach high altitudes. Probable porosity of silicate grains results in the smaller density under the same size, which diminishes the acting gravitational force. However, our estimates show that the increase in the porosity of silicate dust is accompanied by the decrease in the radiation pressure efficiency factor, which cumulatively results in insignificant changes in the motion of grains themselves.

Though the rate of the dust loss from a surface unit grows in absolute value with approaching the Galaxy's center, this quantity taken relative to the gas density increases on the Galaxy's periphery. This suggests that the sweeping-out of dust by the stellar radiation pressure may be a factor that influences the formation of the radial gradient in the chemical composition. It was shown that, approaching the Galaxy's edge, the motion of grains becomes oblique to the disc plane, i.e., the radial motion predominates over the vertical one, which may explain the smoothing of the metallicity gradients observed at galactocentric distances of $\sim 10 \mathrm{kpc}$ in many disc galaxies.

The total loss of the Galaxy's mass in the form of dust is $0.03 M_{\odot}$ per year, which considerably influences the chemical evolution of galactic discs. Since the sweeping-out of dust by the stellar radiation pressure is a general mechanism for disc galaxies, it may 
well explain the dust presence in the vicinity of discs and the existence of intergalactic dust. The simulations, within which the rotation of the Galaxy was ignored, also showed that dust grains are effectively swept out. Since this formulation of the problem qualitatively resembles the conditions in elliptic galaxies, our results suggest that circumgalactic dust is likely to be found near such systems.

\section{ACKNOWLEDGMENTS}

We are grateful to the anonymous referee for careful reading of the manuscript and helpful remarks.

\section{FUNDING}

E. Sivkova and B. Shustov acknowledge the support of the Russian Science Foundation (project no. 19-72-20089).

\section{OPEN ACCESS}

This article is licensed under a Creative Commons Attribution 4.0 International License, which permits use, sharing, adaptation, distribution and reproduction in any medium or format, as long as you give appropriate credit to the original author(s) and the source, provide a link to the Creative Commons license, and indicate if changes were made. The images or other third party material in this article are included in the article's Creative Commons license, unless indicated otherwise in a credit line to the material. If material is not included in the article's Creative Commons license and your intended use is not permitted by statutory regulation or exceeds the permitted use, you will need to obtain permission directly from the copyright holder. To view a copy of this license, visit http://creativecommons.org/licenses/by/4.0/.

\section{REFERENCES}

1. E. M. Xilouris, N. D. Kylafis, J. Papamastorakis, E. V. Paleologou, and G. Haerendel, Astron. Astrophys. 325, 135 (1997).

2. E. M. Xilouris, Y. I. Byun, N. D. Kylafis, E. V. Paleologou, and J. Papamastorakis, Astron. Astrophys. 344, 868 (1999); astro-ph/9901158.

3. S. Bianchi, Astron. Astrophys. 471, 765 (2007); arXiv: 0705.1471.

4. G. De Geyter, M. Baes, P. Camps, J. Fritz, I. de Looze, T. M. Hughes, S. Viaene, and G. Gentile, Mon. Not. R. Astron. Soc. 441, 869 (2014); arXiv: 1403.7527.

5. Y. Sofue, Publ. Astron. Soc. Jpn. 39, 547 (1987).

6. J. C. Howk and B. D. Savage, Astron. J. 114, 2463 (1997); astro-ph/9709197.

7. J. C. Howk and B. D. Savage, Astron. J. 117, 2077 (1999); astro-ph/9902061.

8. B. W. Holwerda, W. C. Keel, B. Williams, J. J. Dalcanton, and R. S. de Jong, Astron. J. 137, 3000 (2009).

9. J. C. Howk and B. D. Savage, Astron. J. 119, 644 (2000); astro-ph/9910248.
10. J. Rossa, R.-J. Dettmar, R. A. M. Walterbos, and C. A. Norman, Astron. J. 128, 674 (2004); astro$\mathrm{ph} / 0405401$.

11. T. W. J. Thompson, J. C. Howk, and B. D. Savage, Astron. J. 128, 662 (2004).

12. D. Zaritsky, Astron. J. 108, 1619 (1994).

13. B. Ménard, R. Scranton, M. Fukugita, and G. Richards, Mon. Not. R. Astron. Soc. 405, 1025 (2010); 0902.4240.

14. K.-I. Seon and A. N. Witt, in The Spectral Energy Distribution of Galaxies-SED 2011, Ed. by R. J. Tuffs and C. C. Popescu, IAU Symp. 284, 135 (2012).

15. K.-I. Seon, A. N. Witt, J.-H. Shinn, and I.-J. Kim, Astrophys. J. Lett. 785, L18 (2014); arXiv: 1403.4905.

16. E. Hodges-Kluck and J. N. Bregman, Astrophys. J. 789, 131 (2014); arXiv: 1401.4170.

17. J. A. Irwin and S. C. Madden, Astron. Astrophys. 445, 123 (2006); astro-ph/0509726.

18. J. A. Irwin, H. Kennedy, T. Parkin, and S. Madden, Astron. Astrophys. 474, 461 (2007); arXiv: 0708.3808.

19. R. J. Rand, K. Wood, R. A. Benjamin, and S. E. Meidt, Astrophys. J. 728, 163 (2011); arXiv: 1101.1491.

20. S. K. Sethi, Y. Shchekinov, and B. B. Nath, Astrophys. J. Lett. 850, L20 (2017); arXiv: 1711.00476.

21. M. Bocchio, S. Bianchi, L. K. Hunt, and R. Schneider, Astron. Astrophys. 586, A8 (2016); arXiv: 1509.07677.

22. A. Abergel, P. A. R. Ade, N. Aghanim, M. I. R. Alves, G. Aniano, C. Armitage-Caplan, M. Arnaud, M. Ashdown, F. Atrio-Barand ela, et al. (Planck Collab.), Astron. Astrophys. 571, A11 (2014); arXiv: 1312.1300.

23. E. Hodges-Kluck, L. Corrales, S. Veilleux, J. Bregman, J. Li, and M. Melendez, Bull. Am. Astron. Soc. 51, 249 (2019); arXiv: 1903.09693.

24. H. Hirashita and C.-Y. Lin, Planet. Space Sci. (2018).

25. R. J. Rand, K. Wood, and R. A. Benjamin, Astrophys. J. 680, 263 (2008); arXiv: 0802.3156.

26. H. Kaneda, M. Yamagishi, T. Suzuki, and T. Onaka, Astrophys. J. Lett. 698, L125 (2009); arXiv: 0905.3800.

27. H. Kaneda, D. Ishihara, T. Suzuki, N. Ikeda, T. Onaka, M. Yamagishi, Y. Ohyama, T. Wada, and A. Yasuda, Astron. Astrophys. 514, A14 (2010); arXiv: 1002.4521.

28. A. McCormick, S. Veilleux, and D. S. N. Rupke, Astrophys. J. 774, 126 (2013).

29. A. McCormick, S. Veilleux, M. Meléndez, C. L. Martin, J. Bland-Hawthorn, G. Cecil, F. Heitsch, T. Müller, D. S. N. Rupke, and C. Engelbracht, Mon. Not. R. Astron. Soc. 477, 699 (2018); arXiv: 1803.03269.

30. Y.-S. Jo, K.-I. Seon, J.-H. Shinn, Y. Yang, D. Lee, and K.-W. Min, Astrophys. J. 862, 25 (2018); arXiv: 1806.06525.

31. J. C. Pecker, Astron. Astrophys. 18, 253 (1972).

32. R. Y. Chiao and N. C. Wickramasinghe, Mon. Not. R. Astron. Soc. 159, 361 (1972).

33. J. C. Pecker, Astron. Astrophys. 35, 7 (1974).

34. J. M. Greenberg, F. Ferrini, B. Barsella, and S. Aiello, Nature (London, U. K.) 327, 214 (1987).

35. B. Barsella, F. Ferrini, J. M. Greenberg, and S. Aiello, Astron. Astrophys. 209, 349 (1989).

36. A. Ferrara, F. Ferrini, J. Franco, and B. Barsella, Astrophys. J. 381, 137 (1991). 
37. J. I. Davies, P. Alton, S. Bianchi, and M. Trewhella, Mon. Not. R. Astron. Soc. 300, 1006 (1998).

38. E. O. Vasiliev and Y. A. Shchekinov, Astron. Rep. 58, 497 (2014).

39. H. Hirashita and S. Aoyama, Mon. Not. R. Astron. Soc. 482, 2555 (2019); arXiv: 1810.07962.

40. B. M. Shustov and D. Z. Vibe, Astron. Rep. 39, 578 (1995).

41. B. T. Draine, Astrophys. J. 57, 587 (1985).

42. A. Li and B. T. Draine, Astrophys. J. 554, 778 (2001); astro-ph/0011319.

43. H. A. Smith, A. Li, M. P. Li, M. Köhler, M. L. N. Ashby, G. G. Fazio, J. S. Huang, M. Marengo, Z. Wang, S. Willner, et al., Astrophys. J. 716, 490 (2010); arXiv: 1004.2277.

44. D. Semenov, T. Henning, C. Helling, M. Ilgner, and E. Sedlmayr, Astron. Astrophys. 410, 611 (2003); astroph/0308344.

45. K. U. Allen, Astrophysical Quantities (Athlone Press, London, 1973).

46. A. Cox, Allen's Astrophysical Quantities, 4th ed. (AIP, New York, 2000). https://cds.cern.ch/record/441599

47. P. J. McMillan, Mon. Not. R. Astron. Soc. 414, 2446 (2011); arXiv: 1102.4340.

48. I. N. Reid, J. E. Gizis, and S. L. Hawley, Astron. J. 124, 2721 (2002).

49. J. Bovy, Mon. Not. R. Astron. Soc. 470, 1360 (2017); arXiv: 1704.05063.

50. J. F. Navarro, C. S. Frenk, and S. D. M. White, Astrophys. J. 490, 493 (1997); astro-ph/9611107.

51. I. Chattopadhyay, M. Sharma, B. B. Nath, and D. Ryu, Mon. Not. R. Astron. Soc. 423, 2153 (2012); arXiv: 1204.1133.

52. F. Nesti and P. Salucci, J. Cosmol. Astropart. Phys. 2013, 016 (2013); arXiv: 1304.5127.

53. B. T. Draine and E. E. Salpeter, Astrophys. J. 231, 77 (1979).

54. P. M. W. Kalberla and J. Kerp, Ann. Rev. Astron. Astrophys. 47, 27 (2009).

55. A. Gupta and S. Sahijpal; arXiv: 2004.11328 (2020).

56. R. Gehrz, in Interstellar Dust, Ed. by L. J. Allamandola and A. G. G. M. Tielens, IAU Symp. 135, 445 (1989).

57. A. P. Jones, L. Fanciullo, M. Köhler, L. Verstraete, V. Guillet, M. Bocchio, and N. Ysard, Astron. Astrophys. 558, A62 (2013); arXiv: 1411.6293.

58. M. Matsuura, M. J. Barlow, A. A. Zijlstra, P. A. Whitelock, M. R. L. Cioni, M. A. T. Groenewegen, K. Volk, F. Kemper, T. Kodama, E. Lagadec, et al., Mon. Not. R. Astron. Soc. 396, 918 (2009); arXiv: 0903.1123.

59. A. Nanni, M. A. T. Groenewegen, B. Aringer, S. Rubele, A. Bressan, J. T. van Loon, S. R. Goldman, and
M. L. Boyer, Mon. Not. R. Astron. Soc. 487, 502 (2019); arXiv: 1904.06702.

60. R. McKinnon, P. Torrey, and M. Vogelsberger, Mon. Not. R. Astron. Soc. 457, 3775 (2016); arXiv: 1505.04792.

61. S. Zhukovska, T. Henning, and C. Dobbs, Astrophys. J. 857, 94 (2018); arXiv: 1803.01929.

62. N. C. Wickramasinghe, Mon. Not. R. Astron. Soc. 159, 269 (1972).

63. T. Jackson, Ž. Ivezic, and G. R. Knapp, Mon. Not. R. Astron. Soc. 337, 749 (2002); astro-ph/0202407.

64. A. Siebert, O. Bienaymé, and C. Soubiran, Astron. Astrophys. 399, 531 (2003), astro-ph/0211328.

65. F. Bresolin, R. C. Kennicutt, and E. Ryan-Weber, Astrophys. J. 750, 122 (2012); arXiv: 1203.0956.

66. S. M. Andrievsky, R. E. Luck, P. Martin, and J. R. D. Lépine, Astron. Astrophys. 413, 159 (2004).

67. K. Z. Arellano-Córdova, C. Esteban, J. García-Rojas, and J. E. Méndez-Delgado, Mon. Not. R. Astron. Soc. 496, 1051 (2020); arXiv: 2005.11372.

68. U. J. Sofia, J. A. Cardelli, and B. D. Savage, Astrophys. J. 430, 650 (1994).

69. X. Wang, T. A. Jones, and T. Treu, AAS Meeting Abstr. 233, 206.02 (2019).

70. J. L. Hou, N. Prantzos, and S. Boissier, Astron. Astrophys. 362, 921 (2000); astro-ph/0007164.

71. K. Cunha, P. M. Frinchaboy, D. Souto, B. Thompson, G. Zasowski, C. Allende Prieto, R. Carrera, C. Chiappini, J. Donor, D. A. García-Hernández, et al., Astron.e Nachr. 337, 922 (2016); arXiv: 1601.03099.

72. R. E. Luck and D. L. Lambert, Astron. J. 142, 136 (2011); arXiv: 1108.1947.

73. A. Shukurov, L. F. S. Rodrigues, P. J. Bushby, J. Hollins, and J. P. Rachen, Astron. Astrophys. 623, A113 (2019); arXiv: 1809.03595.

74. M. S. Murga, D. S. Wiebe, E. E. Sivkova, and V. V. Akimkin, Mon. Not. R. Astron. Soc. 488, 965 (2019); arXiv: 1906.11308.

75. L. Casagrande, V. Silva Aguirre, K. J. Schlesinger, D. Stello, D. Huber, A. M. Serenelli, R. Schönrich, S. Cassisi, A. Pietrinferni, S. Hodgkin, et al., Mon. Not. R. Astron. Soc. 455, 987 (2016); arXiv: 1510.01376.

76. H. J. Rocha-Pinto, J. Scalo, W. J. Maciel, and C. Flynn, Astron. Astrophys. 358, 869 (2000); astro-ph/0001383.

77. M. Cignoni, S. Degl'Innocenti, P. G. Prada Moroni, and S. N. Shore, Astron. Astrophys. 459, 783 (2006); astro-ph/0608654.

Translated by E. Petrova 